\title{
Clozapine-Induced Pericarditis
}

\author{
J Markovic', T Momcilov-Popin², D Mitrovic'1, S Ivanovic-Kovacevic', S Sekuli³, A Stojsic-Milosavljevic ${ }^{4}$ \\ ${ }^{1}$ Center for Child and Adolescent Psychiatry, Institute of Psychiatry, Clinical Center of Vojvodina; Faculty of Medicine, University of Novi \\ Sad, Novi Sad, Serbia \\ ${ }^{2}$ Head, Department of Heart Defects, Clinic of Cardiology, Institute of Cardiovascular Diseases of Vojvodina; Faculty of Medicine, \\ University of Novi Sad, Novi Sad, Serbia \\ ${ }^{3}$ Institut of Neurology, Clinical Center of Vojvodina; Faculty of Medicine, University of Novi Sad, Novi Sad, Serbia \\ 4Department for Echocardiography, Clinic of Cardiology, Institute of Cardiovascular Diseases of Vojvodina; Faculty of Medicine, \\ University of Novi Sad, Novi Sad, Serbia
}

\begin{abstract}
Objective: To report a case of a patient treated with clozapine who developed pericarditis with pericardial effusion that resolved when the drug was discontinued. Method: Case report of a 21 -year-old man with psychotic disorder that had been stable on clozapine therapy for five months (after failure of atypical antipsyhotic agents) presented to the emergency department complaining of chest pain and progressive shortness of breath that had lasted for a few days. Echocardiography showed a pericardial effusion suggestive of a cardiac tamponade, and the fluid was removed by pericardiocentesis. All other possible causes of the pericardial effusion were ruled out and clozapine was suspected as the most likely explanation. Clozapine was discontinued and the patient's symptoms improved markedly. Discussion: According to the Naranjo probability scale, clozapine is a probable cause of pericarditis. Although clozapine is a known cause of myocarditis and cardiomyopathy, there are only several reports in the literature describing clozapine-induced pericarditis and pericardial effusion. In our patient, the pericardial effusion cleared within several days following clozapine discontinuation. Conclusion: There have been only a few cases of clozapine-induced pericarditis reported in the literature, however this adverse effect of clozapine can occur, as this case report clearly demonstrates. Cardiac adverse effects of clozapine are potentially life threatening, hence early recognition is essential to prevent serious outcomes.
\end{abstract}

Key words: Clozapine; Pericarditis; Pericardial effusion; Adverse reaction

Received: 03-03-2010

Accepted: $28-06-2010$

doi: http://dx.doi.org/10.4314/ajpsy.v14i3.7

\section{Introduction}

Clozapine is a drug of choice in schizophrenia when two or more other therapies have failed, a situation which occurs in around $30 \%$ of patients. ${ }^{1}$ Clinical trial data indicate that it is more effective than other antipsychotic agent ${ }^{2,3}$ and is associated with a 6 -fold reduction in the number of suicides in schizophrenics. ${ }^{4,5}$

Orthostatic hypotension and tachycardia are wellrecognized cardiovascular adverse effects of clozapine, occurring in up to $9 \%$ and $25 \%$ of patients respectively, but are rarely clinically important. ${ }^{6,7}$ The increasing number of more serious clozapine-related cardiac complications (myocarditis, pericarditis and cardiomyopathy) reported in

Correspondence

Dr. J Markovic

Institute of Psychiatry, Center for Child and Adolescent Psychiatry,

Clinical Center of Vojvodina. Hajduk Velikova 7, 21000 Novi Sad, Serbia

email: tektonika@nscable.net the literature have given rise to concerns about the risk of acute cardiac adverse reactions in patients treated with the drug. The chief clinical symptoms described were shortness of breath, heart palpitations/pains, diminished endurance, and thoracic pains. In the majority of cases,

electrocardiographic changes, pericardial effusion, pleural effusion and nonspecific signs of inflammation were found.

We report a case of pericarditis associated with clozapine treatment. To our knowledge, there are only a few reports of clozapine-induced pericarditis and pericardial effusion in the literature to date. Wehmeier et al. ${ }^{8}$ reported, in their review of the literature, that they found 65 cases of myocarditis, 52 cases of cardiomyopathy and only 6 cases of pericarditis occurring during clozapine treatment.

\section{Case report}

A 21 -year-old man with a psychotic disorder that had been stable on clozapine therapy for five months (after failure of atypical antipsyhotic agents) presented to the emergency 
department complaining of chest pain and progressive shortness of breath that had lasted for a few days.

The patient had been diagnosed with psychotic disorder one year previously and had been stable on clozapine therapy (100mg in the morning and 100mg in the evening) for five months before the onset of cardiac symptoms. His only other medication was sertraline 50mg/day, which he had been taking for four months. His medical history was otherwise insignificant. The patient denied any substance use except cigarette smoking (10-15 cigarettes per day).

On physical examination, fever $\left(\max t-37.2^{\circ} \mathrm{C}\right)$, tachycardia (heart rate 110 beats/min) and dyspnea were the only positive findings. Electrocardiography showed sinus tachycardia and chest radiography showed increased dimensions of cardiac shadow with an enlarged left ventricle. The levels of C-reactive protein (120.5 mg/L) and fibrinogen (83 g/L) were elevated, and sedimentation rate was increased (max. $98 \mathrm{~mm} / \mathrm{h}$ ). Other laboratory tests (complete blood count, T3, T4, TSH, urinalysis) where within normal limits, and the tests for antinuclear antibodies, anti-heart antibodies, C3, $\mathrm{C} 4$, procalcitonin and the urine toxicology screen were negative

Echocardiography (Figure 1) showed a pericardial effusion suggestive of a cardiac tamponade. The patient was urgently transferred to the coronary emergency unit, where more than $600 \mathrm{ml}$ of serosanguinous fluid was removed by pericardiocentesis. The standard therapy, including antibiotic and anti-rheumatic medication was administered, the patient denied any subjective symptoms, so he was transferred to a general cardiology department.

The following day, the patient had the same subjective symptoms, including dyspnea and tachycardia, upon which he was urgently transferred back to the coronary emergency unit, where echocardiography showed gradual fluid reaccumulation. The patient received two different classes of antibiotics, to which he responded with moderate reduction in subjective symptoms. On day 6, echocardiography showed no significant changes in the quantity of fluid despite the antibiotic and anti-rheumatic therapy. An extensive evaluation for possible infectious, metabolic and ischemic etiologies did not reveal a convincing cause.

\section{Figure 1: Echocardiogram showing a pericardial effusion around the heart}

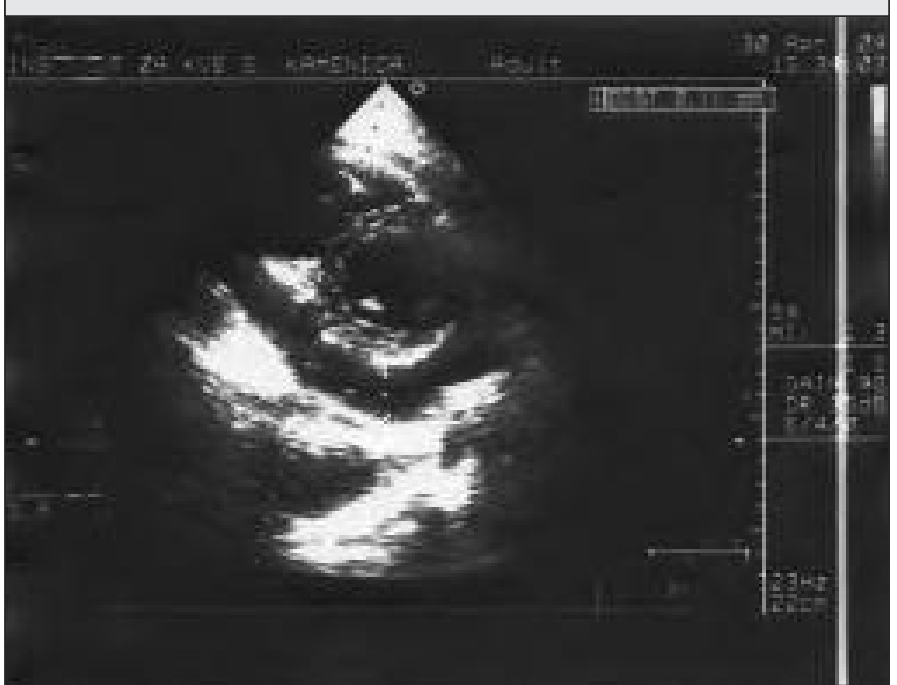

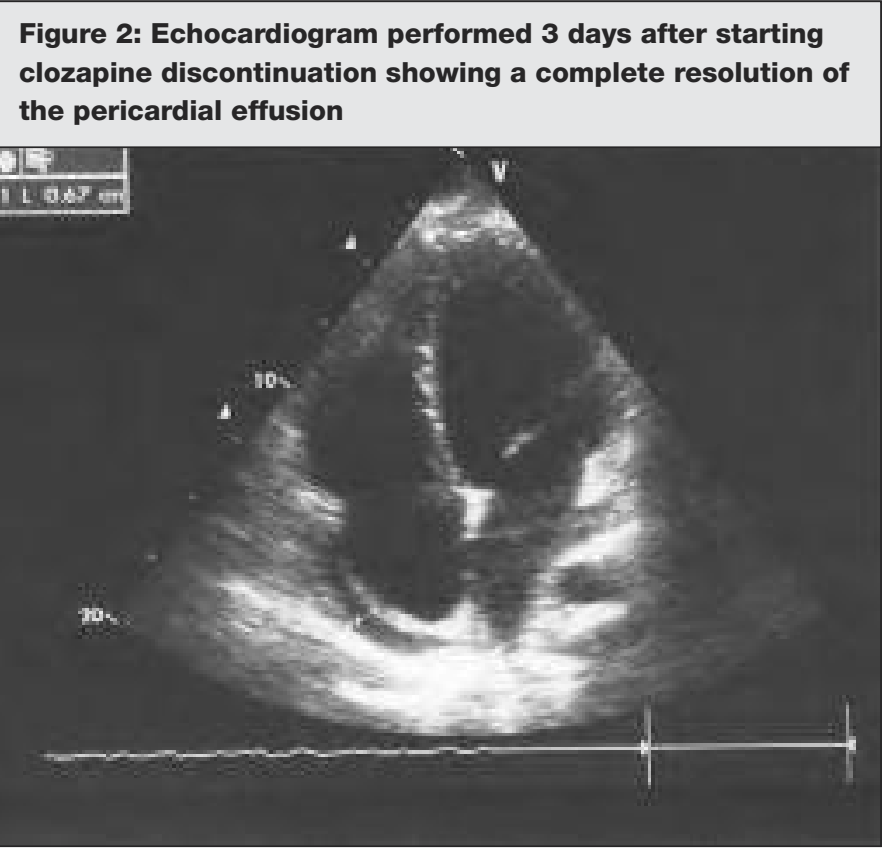

The consulting psychiatrist suggested clozapine as a possible cause of the effusion, and it was decided to gradually replace clozapine with risperidone. Within the following three days the patient's symptoms improved markedly and echocardiography performed on day 20 showed only small amounts of residual fluid (Figure 2). The patient's symptoms continued to improve and ten days later he was discharged. At 3-month follow-up, there was no reaccumulation of pericardial fluid, and transthoracic echocardiography showed a complete resolution of pericardial effusion.

\section{Discussion}

There are a few possible mechanisms to explain clozapineinduced cardiac adverse reactions, however the details of the molecular mechanisms by which myocarditis, pericarditis and cardiomyopathy are precipitated are still far from clear. However, the fact that there is a strong relationship between clozapine and myocarditis or cardiomyopathy is beyond doubt. 8,9

The clinical presentation of clozapine-induced pericarditis may range from a fulminating one resulting in death within hours to a mild influenza-like illness where the presence of pericarditis is suggested by the onset of tachycardia and increased levels of troponin I and/or T. Therefore, a high level of diagnostic suspicion is needed, since sudden death in patients with drug-resistant schizophrenia may otherwise be wrongly attributed to suicide, drug-induced arrhythmia, drug overdose, or other unknown cause. ${ }^{10}$ On the other hand, features such as fever ${ }^{11}$, tachycardia and eosinophilia ${ }^{12}$ commonly resemble the symptom complex that accompanies the initiation of clozapine dosing.

Our case of pericarditis is most likely iatrogenic, which is supported by the following observations. First, the patient lacked cardiac risk factors, and symptomatic pericarditis is uncommon in physically healthy young people. Second, other etiological factors that could have caused pericarditis were excluded. Third, the signs and symptoms resolved 
after discontinuation of the offending agent, clozapine.

Although the patient was receiving both clozapine and sertraline when he developed pericarditis, implicating sertraline is less convincing. A literature search for association between antipsychotics and pericarditis yields almost exclusively cases of clozapine-induced disease. Chlorpromazine, fluphenazine, haloperidol and risperidone have also been implicated, however a true association warrants further investigation for these agents. ${ }^{13}$

The fact that pericardial fluid reaccumulated shortly after the first pericardiocentesis, while the patient continued taking clozapine, also supports the possibility of a clozapineinduced pericardial effusion. The Naranjo probability scale also suggested a probable relationship between clozapine use and pericariditis with effusion. ${ }^{14}$

\section{Conclusion}

Before initiating clozapine, an appropriate medical history and physical examination (including a screening for possible underlying cardiac problems) is advisable. A family history including questions pertaining to premature coronary heart disease and schizophrenia with an associated cardiac problem should be assessed. ${ }^{15}$ Although there have been only a few cases of clozapine-induced pericarditis reported, this adverse reaction may occur, as this case report demonstrates. Clozapine-induced cardiac adverse reactions may be life threatening, and early recognition are therefore essential to prevent serious outcomes.

\section{References}

1. Kilian JG, Kerr K, Lawrence C, Celermajer DS. Myocarditis and cardiomyopathy associated with clozapine. Lancet 1999;354(9193):1841-5

2. Freedman R. The choice of antipsychotic drugs for schizophrenia. N Engl J Med 2005;353(12):1286-8.
3. Lewis SW, Barnes TR, Davies L, Murray RM, Dunn G, Hayhurst KP, et al. Randomized controlled trial of effect of prescription of clozapine versus other second-generation antipsychotic drugs in resistant schizophrenia. Schizophr Bull 2006;32(4):715-23.

4. Walker AM, Lanza LL, Arellano F, et al. Mortality in current and former users of clozapine. Epidemiology 1997;8:671-7.

5. Green A, Burgees E. Use in schizophrenia: effects of clozaine vs. risperidone. Shizophr Res 2003;60:81-5

6. Fitzsimons J, Berk M, Lambert T, et al. A review of clozapine safety. Expert Opin Drug Saf 2005;4: 731-44.

7. Layland JJ, Liew D, Prior DL. Clozapine-induced cardiotoxicity: a clinical update. Med J Aust 2009; 19: 190-2

8. Wehmeier PM, Heiser P, Remschmidt H. Myocarditis, pericarditis and cardiomyopathy in patients treated with clozapine. J Clin Pharm Ther 2005; 30: 91-6

9. Raju P, Rao GK, Davis G. Pericardial effusion in patients with schizophrenia: are they on clozapine? Emerg Med J 2008:25:383-4.

10. Joukamaa M, Heliovaara M, Knekt P, Aromaa A, Raitasalo R, Lehtinen V. Schizophrenia, neuroleptic medication and mortality. Br J Psychiatry 2006; 188:122-7.

11. Merrill DB, Ahmari SE, Bradford J-ME, Lieberman JA. Myocarditis during clozapine treatment. Am J Psychiatry 2006;1 63(2):204-8.

12. Chatterton R. Eosinophilia after commencement of clozapine treatment. Aust N Z J Psychiatry 1997;31 (6):874-6

13. Coulter D, Bate A, Meyboom R, et al. Antipsychotic drugs and heart muscle disorder in international pharmacovigilnace: data mining study. BMJ 2001;322:1207-9.

14. Naranjo CA, Busto U, Sellers EM, et al. A method for estimating the probability of adverse drug reactions. Clin Pharmacol Ther 1981;30:239-45

15. Pastor CA, Mehta M. Masked clozapine-induced cardiomyopathy. JABFM 2008; 21 (1): 70-4

16. Rathore S, Masani ND, Callaghan PO. Clozapine-induced effusoconstrictive pericarditis. Cardiology 2007; 108:183-5.

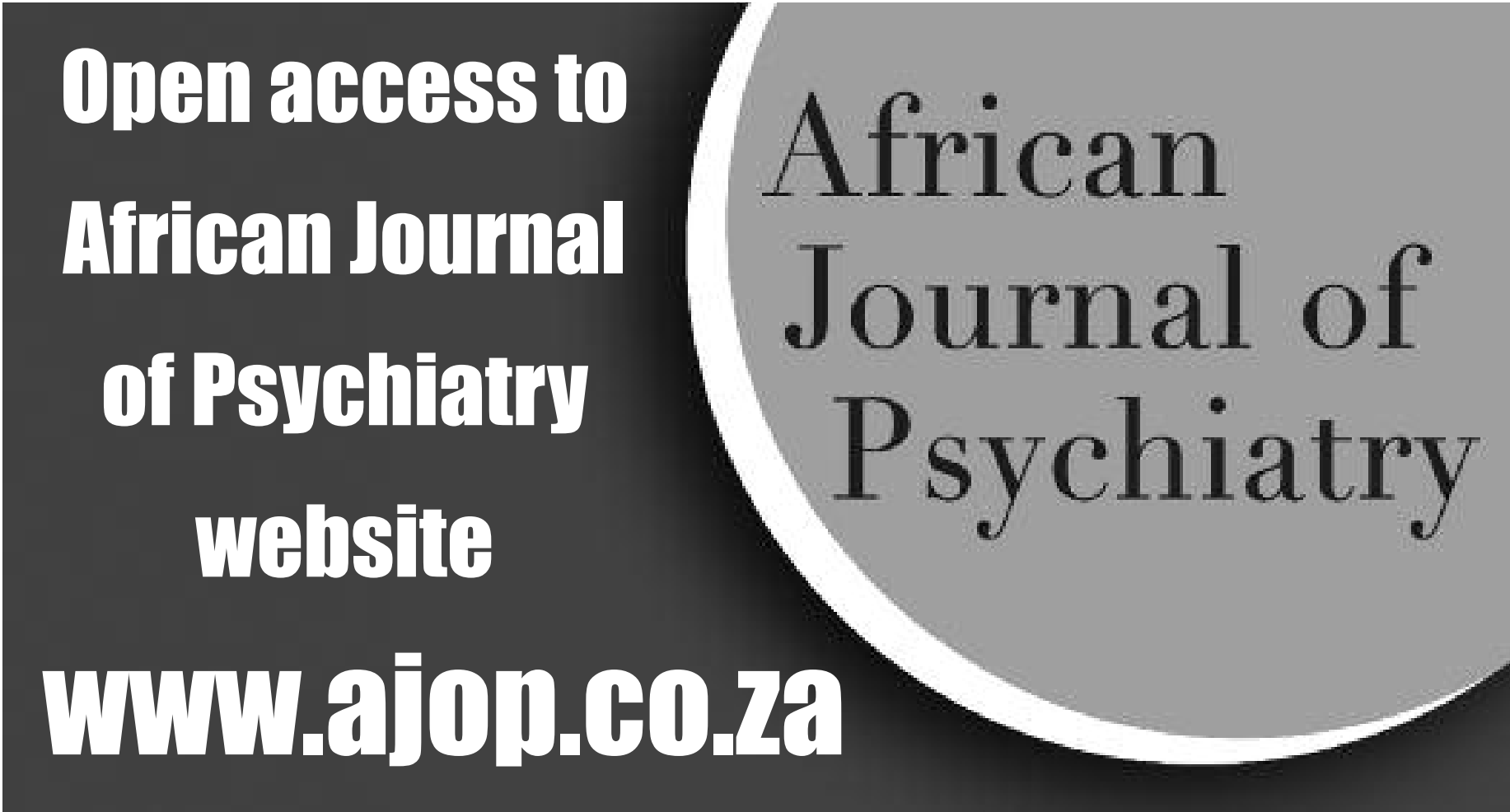

